\title{
Tricuspid valve regurgitation after heart transplantation
}

\author{
Murray H. Kwon, Richard J. Shemin \\ Division of Cardiac Surgery, David Geffen School of Medicine at UCLA, Los Angeles, CA, USA \\ Correspondence to: Richard J. Shemin. Department of Surgery, Cardiovascular Center, David Geffen School of Medicine at UCLA, 100 UCLA Med \\ Plaza Suite 730, Los Angeles, CA 90095, USA. Email: Rshemin@mednet.ucla.edu.
}

\begin{abstract}
Tricuspid valve regurgitation (TVR) in the orthotopic heart transplant (OHT) recipient is quite common and has varied clinical sequelae. In its severest forms, it can lead to right-sided failure symptoms indistinguishable from that seen in native heart TVR disease. While certain implantation techniques are widely recognized to reduce the risk of TVR in the cardiac allograft, concomitant tricuspid annuloplasty, while having advocates, is not currently accepted as a routinely established adjunct. Decisions to surgically correct TVR in the OHT recipient must be made carefully, as certain clinical scenarios have high risk of failure. Like in the native heart, anatomic etiologies typically have the greatest chances for success compared to functional etiologies. While repair options have been utilized, there is emerging data to support replacement as the more durable option. While mechanical prostheses are impractical in the heart transplant recipient, biologic valves offer the advantage of continued access to the right ventricle for biopsies in addition to acceptable durability in the low pressure system of the right side.
\end{abstract}

Keywords: Heart transplantation; tricuspid valve regurgitation (TVR) cardiac biopsy; tricuspid value repair

Submitted Feb 26, 2016. Accepted for publication Apr 04, 2017.

doi: $10.21037 /$ acs.2017.04.02

View this article at: http://dx.doi.org/10.21037/acs.2017.04.02

\section{Introduction}

Orthotopic heart transplantation (OHT) remains the gold standard therapy for advanced heart failure refractory to medical management. Currently, the number of OHTs performed annually worldwide has remained static at approximately 4,000 , limited mainly by donor organ availability. The median survival of 11 years following OHT has remained consistent throughout time with the major limitation being allograft vasculopathy.

Tricuspid valve regurgitation (TVR) in the donor allograft is the most common valvular complication following heart transplantation with a reported incidence of up to $84 \%$ (1). This high rate likely reflects a liberal application of the diagnosis towards a dynamic process that has many etiologies, ranges of severity, and clinical significance. While the majority of TVR in the posttransplant patient is mild and clinically insignificant, approximately $34 \%$ lies in the moderate to severe range resulting in to decreased quality of life due to symptoms such as peripheral edema, exertional dyspnea, and fatigue (2).
Up to $5.8 \%$ of patients develop refractory symptoms to the extent where surgical correction is warranted (3). It is thus a major cause of morbidity and has an associated mortality quoted as high as $62.5 \%$ (4). These numbers are likely an over-simplification of the impact of TVR on the transplant patient as ultimate symptoms, prognosis, and outlook depend greatly on the etiologies and clinical scenarios leading to the valvular incompetence.

In this article, we will review the etiologies of tricuspid regurgitation in the cardiac transplant allograft, their varied pathophysiologies, and finally surgical indications for repair or replacement.

\section{Etiology of tricuspid regurgitation}

\section{Functional tricuspid regurgitation}

Like the native heart, TVR in the cardiac allograft has both functional and anatomic etiologies. One of the predominant mechanisms of the former is geometric distortion of the tricuspid annulus often influenced at the 
time of transplantation. Shortly after the technique of bicaval anastomosis was introduced by Yacoub and colleagues in 1989 (5), there were multiple single center reports of improved TVR over time when compared to the standard bi-atrial technique $(6,7)$. The enlarged right atrial size of the combined atria in the bi-atrial technique was thought to exacerbate the development of TVR by increasing both wall tension and tricuspid annular size during systole (8). In addition to anatomic distortion, the bi-atrial anastomosis was also thought to lead to asynchronous atrial contractions leading to further dilation over time.

Bi-caval anastomosis alone, however, is not sufficient to prevent the development of TVR. Tension in the bi-caval anastomosis is considered a risk factor for the development of TVR by many implanting surgeons as stretching of the right atrium results in distortion of the tricuspid annulus. One group found that modifying the inferior vena caval anastomosis with augmentation by the donor heart with a flap of the recipient's right atrium leads to a reduction in TVR to trace or mild in all study participants (9). The issue with many of these single center studies, however, is that they were underpowered and failed to demonstrate protection from TVR at time periods longer than 1 month (10). A more recent review of the United Network for Organ Sharing database failed to demonstrate a survival benefit following comparison with logistic regression and a proportional hazard model between patients transplanted using the bi-caval versus bi-atrial technique (11).

Functional TVR is also due to progressive annular dilation. Like its left side atrioventricular cousin, this is predominantly a problem with the right ventricle rather than a valvular issue which has obvious implications for subsequent repair. RV failure, for example, can result from high pulmonary vascular resistance. While reversible pulmonary hypertension $(\mathrm{PH})$ has not been associated with adverse transplant outcomes, fixed $\mathrm{PH}$ remains a contraindication for this therapy mainly due to the resultant right sided volume and pressure overload. Echocardiographic assessment of patients with tricuspid regurgitation and $\mathrm{PH}$ demonstrates that the RV not only dilates, but also increases in length along the superior-inferior axis leading to valvular tethering and reduced coaptation (12). Tricuspid regurgitation in these settings is a particularly vexing conundrum as repair or replacement options that fix the regurgitation may do little to influence overall RV function. While re-transplantation is considered in these situations, there may be little to support the idea that the new heart will behave any differently than the original when faced with ongoing elevated pulmonary vascular resistances.
Perhaps the most encouraging data revolves around remodeling of the pulmonary circuit pre-transplant with mechanical circulatory support devices of the left ventricle. When bridged to transplant with left ventricular assist devices (LVADs), patients with what was once thought to be fixed $\mathrm{PH}$, albeit due to left-sided failure, had similar post-transplant outcomes to those without PH (13). Indeed, by offsetting pulmonary vascular resistance, it has been observed that LVADs improve RV function, thereby minimizing TVR and obviating the need for concomitant tricuspid valve repair (14).

The presence of intraoperative TVR of moderate or greater severity adversely impacts long term survival to the extent that some advocate for concomitant tricuspid valve annuloplasty at the time of heart transplantation (15). A series of 60 heart transplant patients all performed using bi-caval technique were divided into two groups. The first received heart transplant alone while the second group received concomitant DeVega tricuspid annuloplasty. Average length of follow up was approximately 6 years and while there were no differences in survival, the latter group had decreased average TVR $(1.5 \pm 1.3$ vs. $0.5 \pm 0.4$, $\mathrm{P}=0.01$ and a lower percentage of $2+$ or greater TVR $(34 \%$ vs. $0 \%, \mathrm{P}=0.01)(16)$. The authors assert that given these advantages, that concomitant tricuspid valve repair should be considered as a routine adjunct to heart transplantation. Many question the durability of the DeVega or any nonrigid annuloplasty procedure, however, in this setting (17). While performing tricuspid annuloplasty on the ex vivo heart is quite straightforward, it is not broadly accepted as standard of care both at our institution or worldwide as there remains clear lack of consensus regarding definitive benefit outside of single center experiences.

Increased episodes of acute rejection greater than International Society for Heart \& Lung Transplantation (ISHLT) Grade 2 was also correlated to increased TVR due to mechanisms of papillary muscle edema and dysfunction as well as asymmetric contractility of the RV (18). Despite resolution of the acute rejection episodes, this study found that TVR increased over the time of surveillance with an incidence of $38 \%$ and $62 \%$ at 1 and 2 years, respectively. When rejection episodes lead to subsequent RV failure there is a significantly higher rate of death and/or retransplantation at 1 year $(\mathrm{OR}=4.80, \mathrm{P}=0.007)(19)$.

\section{Anatomic tricuspid regurgitation}

\section{Biopsy related TVR}

Numerous studies have demonstrated a causal link 
between the number of endomyocardial biopsies (EMB) and the development of TVR. EMB is the current standard of care in routine graft surveillance and typically has a preponderance in the early time period following transplantation. Chordal damage resulting in flail leaflets is the presumed mechanism and development of TVR has been correlated to the number of biopsies performed. While frank leaflet tissue is a rather uncommon finding in EMB specimens, the presence of chordal tissue is a welldocumented phenomenon with one series showing $47 \%$ of patients with new onset TVR having evidence of chordae in their myocardial specimens (20). In another series, there were no cases of severe TVR in patients who have had fewer than 18 biopsies whereas in patients with over 31 procedures, $60 \%$ developed severe TVR (21). The authors' recommendations were thus to configure protocol biopsies to a regimen of less than 31 over the lifetime of the allograft to help reduce the risk of development of severe iatrogenic TVR.

\section{Endocarditis}

While heart transplant recipients are immunosuppressed, the development of endocarditis is a relatively rare event compared to other infections. One review of the literature documented 10 cases out of 659 patients transplanted over an 11-year period. Five of these cases involved the tricuspid valve with a $100 \%$ mortality following diagnosis. Once again, EMB was implicated as a risk factor due to increased susceptibility of the damaged valve to bacterial seeding. Patients with TV endocarditis had a median of 23 EMB compared to patients with mitral valve infection who had only nine. Their review of the literature documented only 22 cases of transplant allograft endocarditis with involvement of the right sided atrio-ventricular valve in seven $(22 \%)$. It is not clear from the paper if valve surgery was attempted in this cohort or if clinical futility prevented this option (22).

\section{Pathophysiology of TVR in the heart transplant recipient}

The pathophysiology of severe tricuspid regurgitation in the transplanted heart is not unlike that which exists in native valve pathology. Loss of coaptation of the tri-leaflet valve whether it be from functional or anatomic etiologies results in regurgitation of blood in systole leading to the combination of elevated right sided pressures as well as decreased forward cardiac output. In the severest forms, the resulting venous hypertension can lead to clinical sequelae of ascites, peripheral edema, and hepato-renal dysfunction. Over time, the pressure and/or volume overload can lead to worsening RV function with a decrement in forward cardiac output that can be difficult to discern from that caused by acute rejection.

Obviously, when functional TVR is present alongside left sided heart disease, symptoms associated with both disease processes may be superimposed such as shortness of breath, exercise intolerance, and overall weakness, malaise, and failure to thrive.

\section{Surgical indications for tricuspid valve repair or replacement}

Multiple studies have shown that the majority of TVR that develops following heart transplantation does not lead to symptoms significant enough to warrant surgical repair $(2,4,6)$. In the minority of patients, however, for whom TVR leads to medically refractory symptoms, repair and replacement options have been employed. Filsoufi and colleagues' study identified 8 out of 138 transplanted patients who required surgery for symptomatic severe TVR. They were divided into two groups of four based on etiology of either annular dilation (functional TVR) or flail leaflet due to biopsy induced chordal rupture (anatomic TVR). Valve repair was attempted in all 8 patients. What was notable was a $50 \%$ failure rate in the anatomic group requiring subsequent reoperation for valve replacement within a 2 -week interval. The functional group also had $50 \%$ failure with repair requiring tricuspid valve replacement, however, this occurred at a longer time period following surgery at 10 months and 4 years, respectively. All patients survived during the follow up period of this study (3). Another data base study performed by the Utah Transplantation Affiliated Hospitals Cardiac Transplant Program found 17 patients over a 20-year interval who required $16 \mathrm{TV}$ replacement and 2 repair procedures due to medically refractory symptoms. A flail leaflet was the cause of TVR in all but one of the patients. There were two mortalities in their series and improvement in symptoms was seen in 12 of the patients following surgery (23).

These studies allude to two important concepts in tricuspid valve surgery in the heart transplant recipient. The first is that like native heart valve disease, indications for surgery may be more straightforward when there is an anatomic valvular issue. In our experience, creating "annular solutions for ventricular problems" that are often the cause 
of functional TVR can be fraught with numerous pitfalls. That is not to say there is no utility in reversing the volume and pressure overload in patients with central TVR due to non-coapting leaflets from annular dilation. However, these patients need to be carefully selected. For example, the combination of intrinsic PH, RV failure, and TVR is particularly ominous and these patients are unlikely to benefit long term from tricuspid valve surgery and carry a high risk for operative mortality in our experience.

The second important point from the data above is the possible advantage of replacement over repair particularly in the anatomic group. Replacement is associated with improved symptoms in surviving patients and seems to have the added benefit of durability when compared to repair options (24). With regards to valve type, mechanical valves would be undesirable due to the inability to subsequently perform EMBs and the need for anticoagulation. The biologic valve, on the other hand, appears to have excellent long-term durability in the low-pressure system of the right heart, does not require anticoagulation, and affords ongoing ability to perform biopsies. There might even be an advantage of low risk of subsequent injury as the chordal apparatus is now taken out of the picture and actual prosthetic valve leaflet injury from EMB is a rare occurrence.

\section{Conclusions}

TVR is a common entity following heart transplant and has varying implications for the patient. In most instances, the degree of severity is mild to moderate and is not associated with clinical sequelae requiring operative intervention. In those cases where medical treatment alone is inadequate, however, symptoms are not unlike that seen in patients with native TVR such as edema, hepato-renal dysfunction, and ascites. It is imperative, however, that careful attention be placed towards proper patient selection. As in native heart valvular disease, the best outcomes are seen with variants of TVR where RV function is still preserved. Great caution must be exercised when assessing functional TVR particularly in the setting of ongoing high pulmonary vascular resistance as this combination makes operative strategies including valve replacement and retransplantation hazardous. Strategies to ameliorate the risk of TVR performed at the time of transplantation such as the construction of bi-caval as well as tension free anastomoses appear to be well established. Concomitant tricuspid valve annuloplasty at the time of OHT, however, while having advocates, is not as yet a commonly accepted practice.

\section{Acknowledgements}

None.

\section{Footnote}

Conflicts of Interest: The authors have no conflicts of interest to declare.

\section{References}

1. Wong RC, Abrahams Z, Hanna M, et al. Tricuspid regurgitation after cardiac transplantation: an old problem revisited. J Heart Lung Transplant 2008;27:247-52.

2. Chan MC, Giannetti N, Kato T, et al. Severe tricuspid regurgitation after heart transplantation. J Heart Lung Transplant 2001;20:709-17.

3. Filsoufi F, Salzberg SP, Anderson CA, et al. Optimal surgical management of severe tricuspid regurgitation in cardiac transplant patients. J Heart Lung Transplant 2006;25:289-93.

4. Berger Y, Har Zahav Y, Kassif Y, et al. Tricuspid valve regurgitation after orthotopic heart transplantation: prevalence and etiology. J Transplant 2012;2012:120702.

5. Yacoub M, Mankad P, Ledingham S. Donor procurement and surgical techniques for cardiac transplantation. Semin Thorac Cardiovasc Surg 1990;2:153-61.

6. Aziz T, Burgess M, Khafagy R, et al. Bicaval and standard techniques in orthotopic heart transplantation: mediumterm experience in cardiac performance and survival. J Thorac Cardiovasc Surg 1999;118:115-22.

7. Laske A, Carrel T, Niederhäuser U, et al. Modified operation technique for orthotopic heart transplantation. Eur J Cardiothorac Surg 1995;9:120-6.

8. Koch A, Remppis A, Dengler TJ, et al. Influence of different implantation techniques on $\mathrm{AV}$ valve competence after orthotopic heart transplantation. Eur J Cardiothorac Surg 2005;28:717-23.

9. Marelli D, Silvestry SC, Zwas D, et al. Modified inferior vena caval anastomosis to reduce tricuspid valve regurgitation after heart transplantation. Tex Heart Inst J 2007;34:30-5.

10. Jacob S, Sellke F. Is bicaval orthotopic heart transplantation superior to the biatrial technique? Interact Cardiovasc Thorac Surg 2009;9:333-42.

11. Davies RR, Russo MJ, Morgan JA, et al. Standard versus 
bicaval techniques for orthotopic heart transplantation: an analysis of the United Network for Organ Sharing database. J Thorac Cardiovasc Surg 2010;140:700-8, 708.e1-2.

12. Topilsky Y, Khanna A, Le Tourneau T, et al. Clinical context and mechanism of functional tricuspid regurgitation in patients with and without pulmonary hypertension. Circ Cardiovasc Imaging 2012;5:314-23.

13. Kanwar M, Raina A, Aponte MP, et al. Pulmonary hypertension in potential heart transplant recipients: current treatment strategies. Curr Opin Organ Transplant 2015;20:570-6.

14. Atluri P, Fairman AS, MacArthur JW, et al. Continuous flow left ventricular assist device implant significantly improves pulmonary hypertension, right ventricular contractility, and tricuspid valve competence. J Card Surg 2013;28:770-5.

15. Anderson CA, Shernan SK, Leacche M, et al. Severity of intraoperative tricuspid regurgitation predicts poor late survival following cardiac transplantation. Ann Thorac Surg 2004;78:1635-42.

16. Jeevanandam V, Russell H, Mather P, et al. Donor tricuspid annuloplasty during orthotopic heart transplantation: long-term results of a prospective controlled study. Ann Thorac Surg 2006;82:2089-95; discussion 2095.

17. Sales VL, McCarthy PM. Durability of functional tricuspid valve repair. Semin Thorac Cardiovasc Surg 2010;22:97-103.

Cite this article as: Kwon MH, Shemin RJ. Tricuspid valve regurgitation after heart transplantation. Ann Cardiothorac Surg 2017;6(3):270-274. doi: 10.21037/acs.2017.04.02
18. Herrmann G, Simon R, Haverich A, et al. Left ventricular function, tricuspid incompetence, and incidence of coronary artery disease late after orthotopic heart transplantation. Eur J Cardiothorac Surg 1989;3:111-7; discussion 118.

19. Haddad F, Fisher P, Pham M, et al. Right ventricular dysfunction predicts poor outcome following hemodynamically compromising rejection. J Heart Lung Transplant 2009;28:312-9.

20. Mielniczuk L, Haddad H, Davies RA, et al. Tricuspid valve chordal tissue in endomyocardial biopsy specimens of patients with significant tricuspid regurgitation. J Heart Lung Transplant 2005;24:1586-90.

21. Nguyen V, Cantarovich M, Cecere R, et al. Tricuspid regurgitation after cardiac transplantation: how many biopsies are too many? J Heart Lung Transplant 2005;24:S227-31.

22. Sherman-Weber S, Axelrod P, Suh B, et al. Infective endocarditis following orthotopic heart transplantation: 10 cases and a review of the literature. Transpl Infect Dis 2004;6:165-70.

23. Alharethi R, Bader F, Kfoury AG, et al. Tricuspid valve replacement after cardiac transplantation. J Heart Lung Transplant 2006;25:48-52.

24. Raghavan R, Cecere R, Cantarovich M, et al. Tricuspid valve replacement after cardiac transplantation. Clin Transplant 2006;20:673-6. 